\title{
A frame work for the detection and Diagnosis of Lung Tumors using Deep learning Methods
}

\author{
P.Jagadeesha ${ }^{a}$ V.S Jayanthi ${ }^{b}$, and Radhika Bhasker ${ }^{c}$ \\ Assistant Professor, Department of ECE, Saveetha School of Engineering,SIMATS, \\ Chennai-602105,Tamilnadu \\ ${ }^{\mathbf{b}}$ Professor, Department of ECE, Rajagiri School of Engineering \& Technology, Kakkanad, Kochi 682 \\ cAssociate Professor, Department of ECE, Saveetha School of Engineering, \\ SIMATS, Chennai-602105,Tamilnadu
}

Article History: Received: 11 January 2021; Accepted: 27 February 2021; Published online: 5 April 2021

\begin{abstract}
The detection of tumor pixels in lung images is complex task due to its low contrast property. Hence, this paper uses deep learning architectures for both the detection and diagnosis of lung tumors in Computer Tomography (CT) images. In this article, the tumors are detected in lung CT images using Convolutional Neural Networks (CNN) architecture with the help of data augmentation methods. This proposed CNN architecture classifies the lung images into two categories as tumor images and normal images. Then, the segmentation method is used to segment the tumor pixels in the lung CT images and the segmented tumor regions are classified into either mild or severe using proposed $\mathrm{CNN}$ architecture.
\end{abstract}

Keywords: Lung, tumors, deep learning, segmentation, classifications.

\section{Introduction}

The tumor cells are developed in the lung regions due to the genetic reasons and improper food consumption. More alcohol intake is the main reason for the formation of abnormalities in lung regions of the human body [1]. The tumors in the lung regions are identified using different scanning techniques as Computer Tomography (CT) and Positron Emission Tomography (PET). These methods are known as modality techniques or scanning methods [2]. Among this modality of methods, mostly CT images are used to detect the abnormality in lung regions due to its pixel contrast property. The tumor cells in lung CT images are detected using image processing methods such as machine learning algorithms and deep learning algorithms. These artificial intelligence based algorithms requires number of internal and external features from the lung CT images [3-4]. The feature computation is more complex in machine learning algorithms and hence it requires large number of source lung CT images for improving the classification or tumor detection accuracy. These limitations are resolved by implementing the deep learning algorithm for the detection and classifications of lung tumors in lung CT images. Also, the deep learning architectures detected tumor images even in low resolution formats using different data augmentation methods. Hence, this paper uses deep learning architectures for both the detection and diagnosis of lung tumors in CT images.

Fig.1 (a) shows the lung image using CT modality method and Fig.1 (b) shows the lung image using PET modality method.

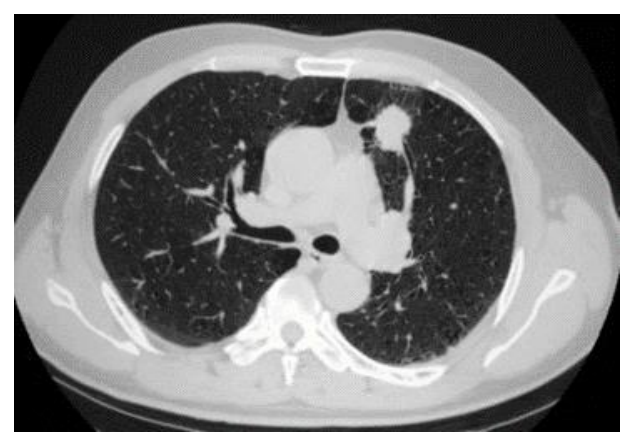

(a)

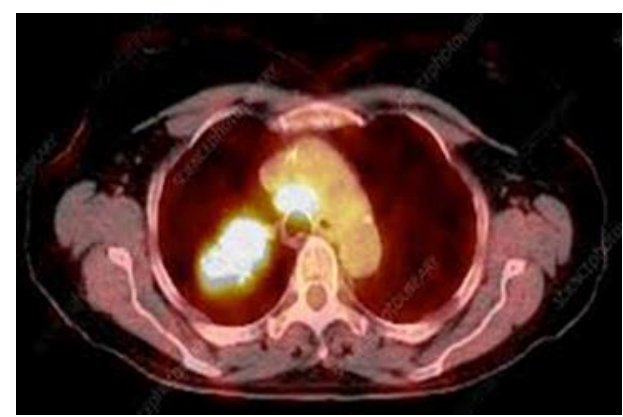

(b)

Figure 1 (a) Lung CT image (b) Lung PET image

\section{Literature survey}

Sori et al. (2020) used Denoising First with Deep learning Networks (DFD-Net) for the detection and differentiation of lung CT images into various category of images. The authors initially applied Deep Residual learning denoising model (DR-Net) for the identification of noises in the lung CT images and then filter was applied on the noised images to remove the noise contents. Then, DFD-Net was used to classify the lung CT image into various different categories such as tumor images and normal images. The authors obtained $97.1 \%$ of average tumor diagnosis index, $98.4 \%$ of average segmentation accuracy and $97.8 \%$ of average sensitivity using 
their proposed works. RuoXi Qin et al. (2020) developed multi dimensional attention mechanism for the detection and classifications of lung CT images into either normal or abnormal. The authors computed fined grained features from the lung CT images and these fine grained features were classified using deep learning based multi dimensional attention mechanism in this work. The authors obtained $96.5 \%$ of average tumor diagnosis index, $97.1 \%$ of average segmentation accuracy and $96.9 \%$ of average sensitivity using their proposed works. Lakshmanaprabu et al. (2019) utilized the optimal deep learning algorithm for the detection and classification of tumor affected lung CT images. This method was entirely based on the optimal features which were computed from the source grained lung CT images. These optimal features were data augmented and then classified using developed CNN architecture. The authors obtained $94.7 \%$ of average tumor diagnosis index, $95.6 \%$ of average segmentation accuracy and $96.9 \%$ of average sensitivity using their proposed works.

Teramoto et al. (2017) detected tumor pixels in lung cytological images using deep learning CNN architecture. The non-rugged pixels were detected with the help of the data augmented deep learning architecture which further diagnosed the classified patterns into different diagnosis results. The authors obtained $87.8 \%$ of average tumor diagnosis index, $96.6 \%$ of average segmentation accuracy and $93.9 \%$ of average sensitivity using their proposed works. Sun et al. (2016) used non-linear probabilistic algorithm for the detection of tumor images from non-tumor images. The authors integrated the proposed non-linear probabilistic algorithm with the conventional deep learning architecture to find the tumor pixels in lung CT images. The authors applied different deep learning methods on the source lung CT images to authenticate the effectiveness of these methods for lung tumor classifications. The authors obtained $91 \%$ of average tumor diagnosis index, $93.6 \%$ of average segmentation accuracy and $95.9 \%$ of average sensitivity using their proposed works.

\section{Proposed Methodologies}

In this article, the tumors are detected in lung CT images using CNN architecture with the help of data augmentation methods. This proposed CNN architecture classifies the lung images into two categories as tumor images and normal images. Then, the segmentation method is used to segment the tumor pixels in the lung CT images and the segmented tumor regions are classified into either mild or severe using proposed CNN architecture. The overall proposed flows for lung tumor detection and its classifications using CNN are depicted in Fig.2.

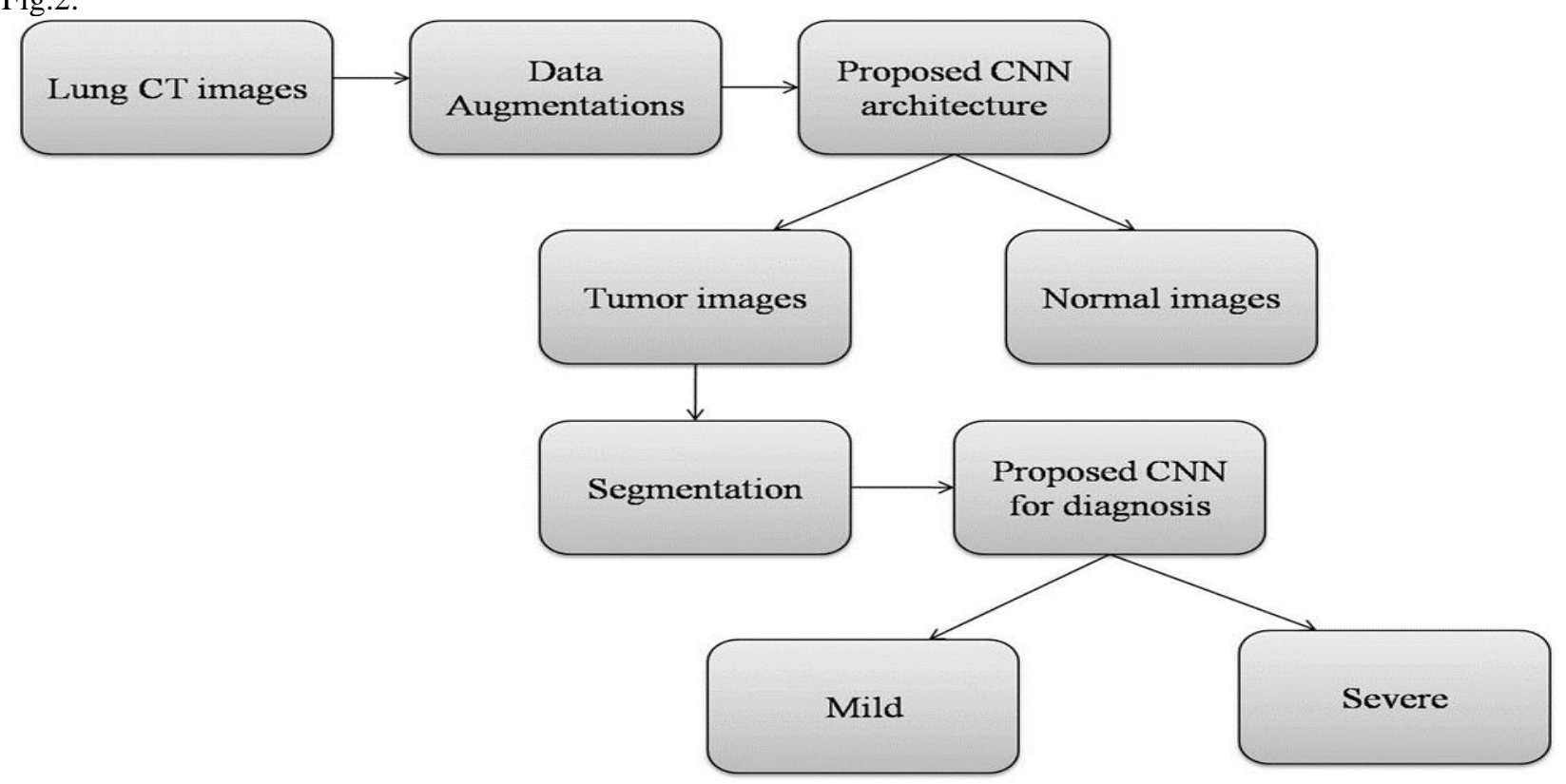

Figure 2 Lung tumor detection and diagnosis- proposed flows

Fig.3 (a) is the lung tumor classifications using proposed CNN and Fig.3(b) is the lung tumor diagnosis using proposed CNN. In this article, ResNet $18 \mathrm{CNN}$ architecture [ ] is used for lung tumor detection and diagnosis purposes.

The lung CT images in the open access dataset are data augmented which is used to increase the image counts for achieving best classification accuracy. In this article, image shifting methods are used as data augmentation methods. In lung tumor classifications as tumor images and normal images, the proposed CNN architecture is designed with 64 Convolutional layers and one pooling layer and two numbers of Fully Connected Neural Networks (FCNN) as illustrated in Fig. 3(a). 
M P.Jagadeesh ${ }^{a}$ V.S Jayanthi $^{b}$, and Radhika Bhasker $^{c}$

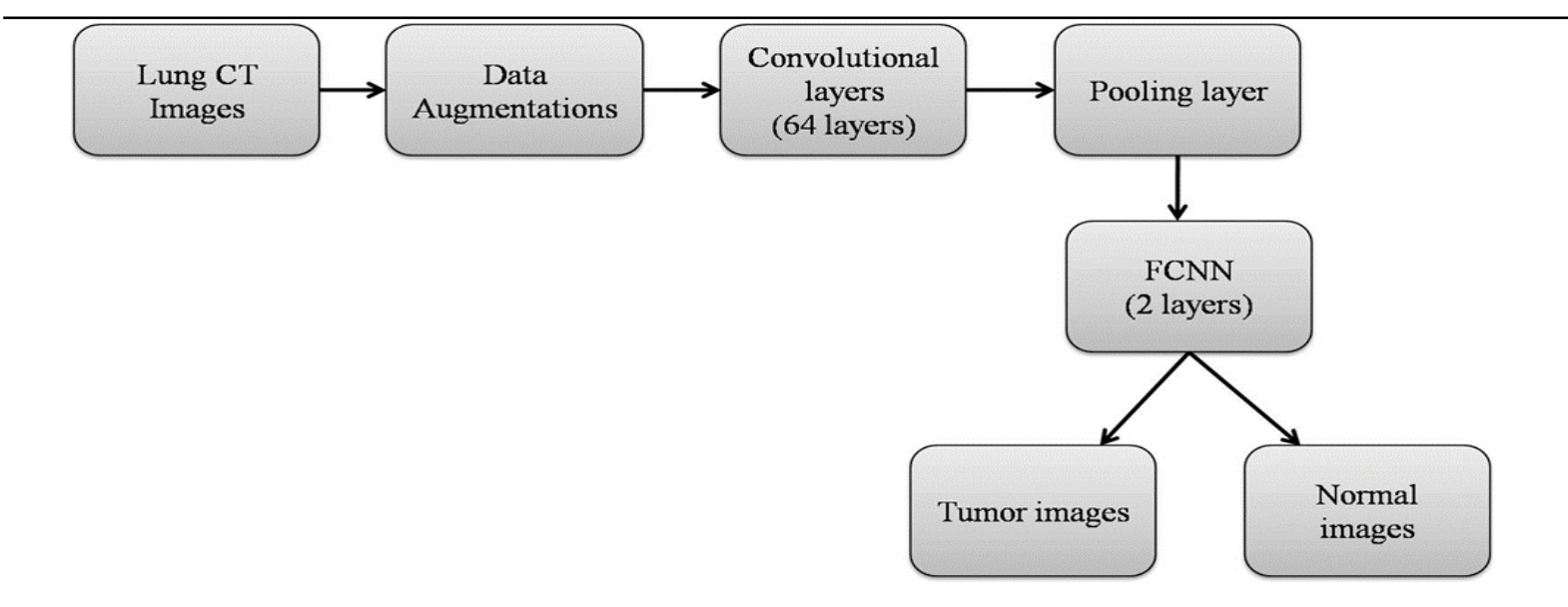

(a)

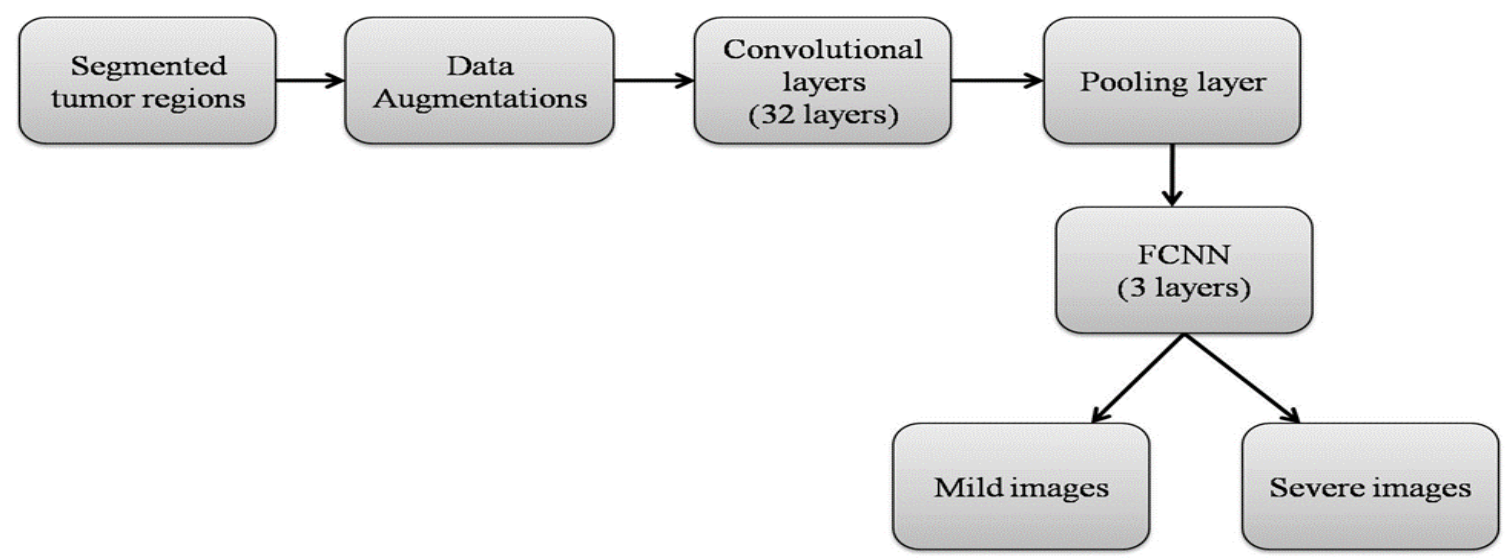

(b)

Figure 3 (a) Lung tumor classifications using proposed CNN (b) Lung tumor diagnosis using proposed CNN

After, the proposed CNN classifies the lung CT images into tumor images and normal images; the watershed segmentation method (Jemimma et al. 2018) is used to segment the tumor pixel regions in classified tumor images. In lung tumor diagnosis as mild images and severe images, the proposed CNN architecture is designed with 32 Convolutional layers and one pooling layer and three numbers of Fully Connected Neural Networks (FCNN) as illustrated in Fig. 3(b).

The classified tumor and normal lung CT images are illustrated in Fig. 4(a) and Fig.4 (b), respectively.

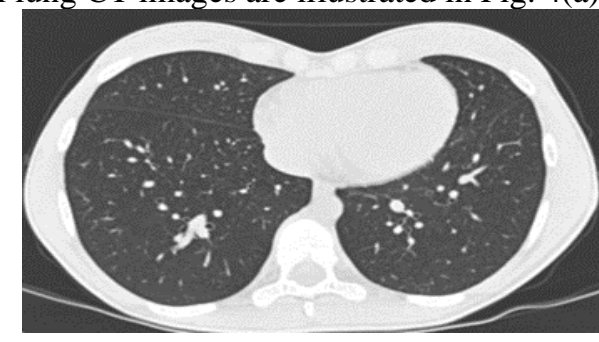

(a)

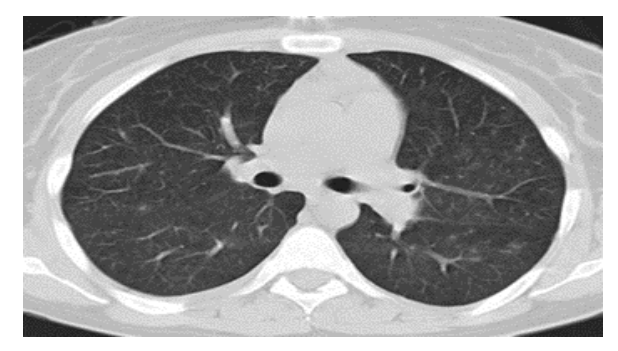

(b)

Figure 4 (a) Normal lung CT image (b) Tumor lung CT image The tumor pixels segmented lung CT images are given in Fig.5. 


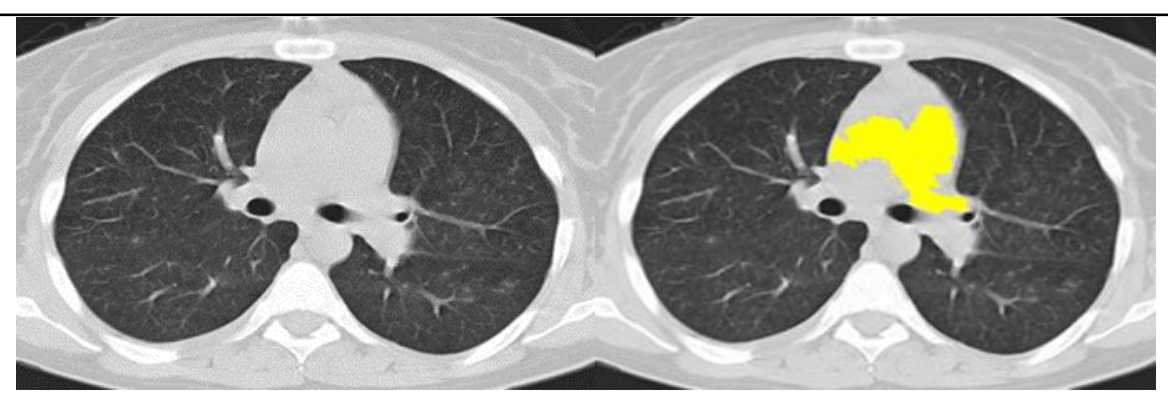

(a) (b)

Figure 5 (a) Tumor affected lung CT image (b) Tumor region segmented image

\section{Results and Discussions}

The proposed lung tumor detection and diagnosis framework in this research work uses Lung Image Database Consortium (LIDC) dataset [11] for evaluating the performance of the system. In this dataset, 888 lung CT images are grouped into two sub categories as normal images which do not have any tumor pixels on it and the tumor affected lung CT images which have the tumor pixels on it. Among these 888 lung CT images, 600 lung CT images are normal images and the remaining 288 lung CT images are abnormal images. These 288 lung abnormal tumor affected CT images are further diagnosed into 'Mild category (200 cases of images) or 'Severe' category ( 88 cases of images) based on the morphological properties of the segmented tumor regions.

This proposed lung CT image classification and diagnosis system is analyzed with the following parameter. The tumor diagnosis index for the mild case of CT lung image is defined by the following equation.

$$
\text { Tumor Diagnosis Index for Mild (TDIM) }=\frac{\text { Tumor detected images as mild }}{\text { Total mild tumor images }} * 100 \%
$$

The tumor diagnosis index for the severe case of CT lung image is defined by the following equation.

$$
\text { Tumor Diagnosis Index for Severe (TDIS) }=\frac{\text { Tumor detected images as severe }}{\text { Total severe tumor images }} * 100 \%
$$

Table 1 is the simulation results in terms of TDIM and TDIS using the proposed framework stated in this article.

Table 1 Simulation results in terms of TDIM and TDIS

\begin{tabular}{|c|c|c|c|}
\hline Lung tumor case & $\begin{array}{c}\text { Experimental } \\
\text { results }\end{array}$ & $\begin{array}{c}\text { Lung tumor } \\
\text { case }\end{array}$ & $\begin{array}{c}\text { Experimental } \\
\text { results }\end{array}$ \\
\hline $\begin{array}{c}\text { Total Mild lung CT } \\
\text { images }\end{array}$ & 200 & $\begin{array}{c}\text { Total severe } \\
\text { lung CT images }\end{array}$ & 88 \\
\hline $\begin{array}{c}\text { Diagnosed mild } \\
\text { lung CT images } \\
\text { using proposed } \\
\text { work }\end{array}$ & 198 & $\begin{array}{c}\text { Diagnosed } \\
\text { severe lung CT } \\
\text { images using } \\
\text { proposed work }\end{array}$ & 87 \\
\hline TDIM in \% & 99 & TDIS in \% & 98.8 \\
\hline
\end{tabular}

TDIM for mild lung images is about $99 \%$ and the TDIS for severe lung images is about $98.8 \%$ in this article.

Also, the concert of the proposed lung tumor diagnosis is evaluated with the help of segmentation accuracy and sensitivity values. The segmentation accuracy is the amount of percentage of the pixels correctly segmented as tumor pixels in lung CT images. The sensitivity is the amount of percentage of the correctly detected tumor and non tumor pixels. Both segmentation accuracy and sensitivity values are used to evaluate the effectiveness of the proposed lung tumor diagnosis work in this article.

Table 2 is the simulation results in terms of segmentation accuracy and sensitivity, with the help of simulation analysis of MATLAB R 2018 version.

\begin{tabular}{|c|c|c|}
\hline \multirow{2}{*}{$\begin{array}{c}\text { Lung } \\
\text { image } \\
\text { sequences }\end{array}$} & \multicolumn{2}{|c|}{ Simulation parameters in \% } \\
\hline & $\begin{array}{l}\text { Segmentation } \\
\text { accuracy }\end{array}$ & Sensitivity \\
\hline 1 & 96.9 & 98.9 \\
\hline 2 & 98.9 & 99.6 \\
\hline 3 & 99.6 & 99.8 \\
\hline
\end{tabular}

Table 2 Simulation results in terms of segmentation accuracy and sensitivity 


\begin{tabular}{|c|l|l|}
\hline 4 & 99.8 & 99.9 \\
\hline 5 & 99.6 & 99.5 \\
\hline 6 & 99.7 & 99.7 \\
\hline 7 & 99.1 & 99.7 \\
\hline 8 & 99.9 & 99.6 \\
\hline 9 & 99.6 & 99.4 \\
\hline 10 & 99.8 & 98.1 \\
\hline
\end{tabular}

Fig.6 is the simulation results of the proposed lung tumor detection system in terms of segmentation accuracy and sensitivity.

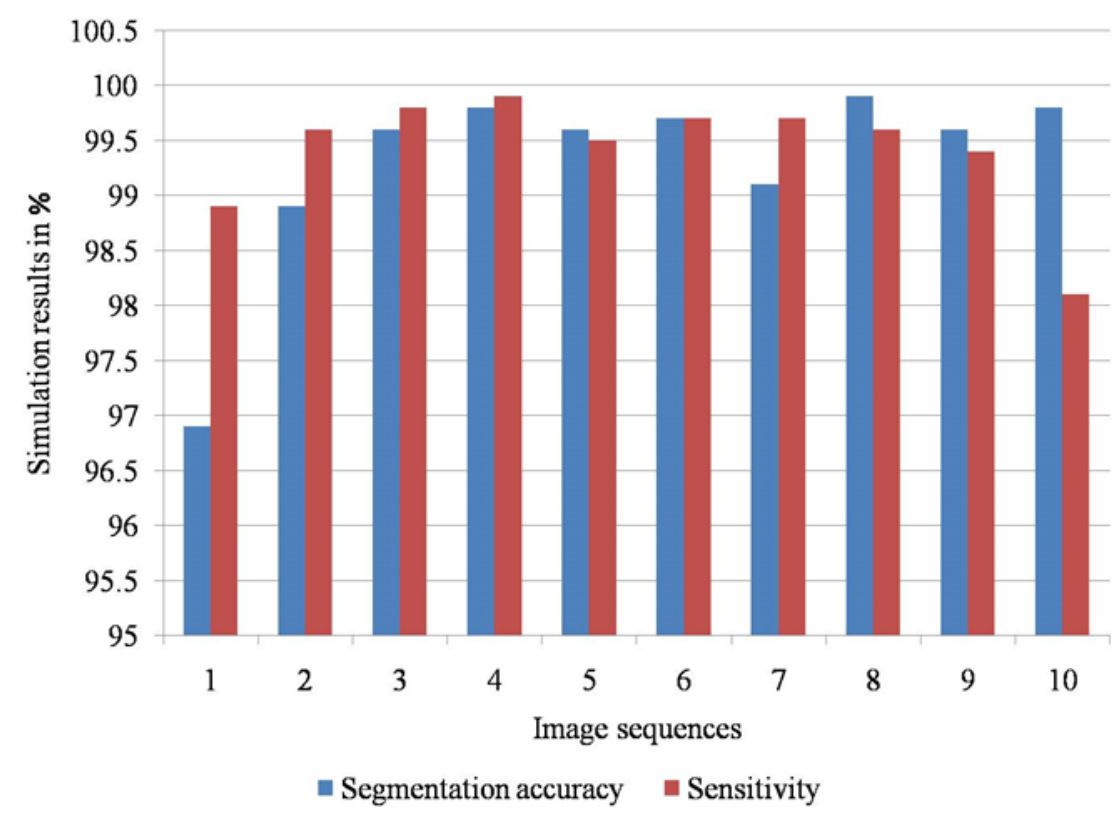

Figure 6 Simulation results of the proposed work

In this article, the proposed lung CT tumor detection system achieves $98.9 \%$ of average diagnosis index, $99.29 \%$ of segmentation accuracy and $99.42 \%$ of sensitivity.

Table 3 Simulation results of the proposed works

\begin{tabular}{|c|c|}
\hline Simulation parameters & Simulation results in \% \\
\hline Average Diagnosis Index & 98.9 \\
\hline Segmentation accuracy & 99.29 \\
\hline Sensitivity & 99.42 \\
\hline
\end{tabular}

The proposed lung tumor detection in lung CT images is compared with the other conventional methods RuoXi Qin et al. (2020) and Sori et al. (2020) in terms of tumor segmentation accuracy, sensitivity and average tumor diagnosis rate, as illustrated in Table 4.

Table 4 Comparisons of the simulation results

\begin{tabular}{|c|c|c|c|}
\hline Methodologies & $\begin{array}{c}\text { Average Diagnosis } \\
\text { Index }\end{array}$ & $\begin{array}{c}\text { Segmentation } \\
\text { accuracy }\end{array}$ & Sensitivity \\
\hline Proposed work & 98.9 & 99.29 & 99.42 \\
\hline RuoXi Qin et al. (2020) & 96.5 & 97.1 & 96.9 \\
\hline Sori et al. (2020) & 97.1 & 98.4 & 97.8 \\
\hline
\end{tabular}

Fig.7 is the graphical illustrations of the comparisons of the proposed work simulation results with existing methods for lung tumor detections. 


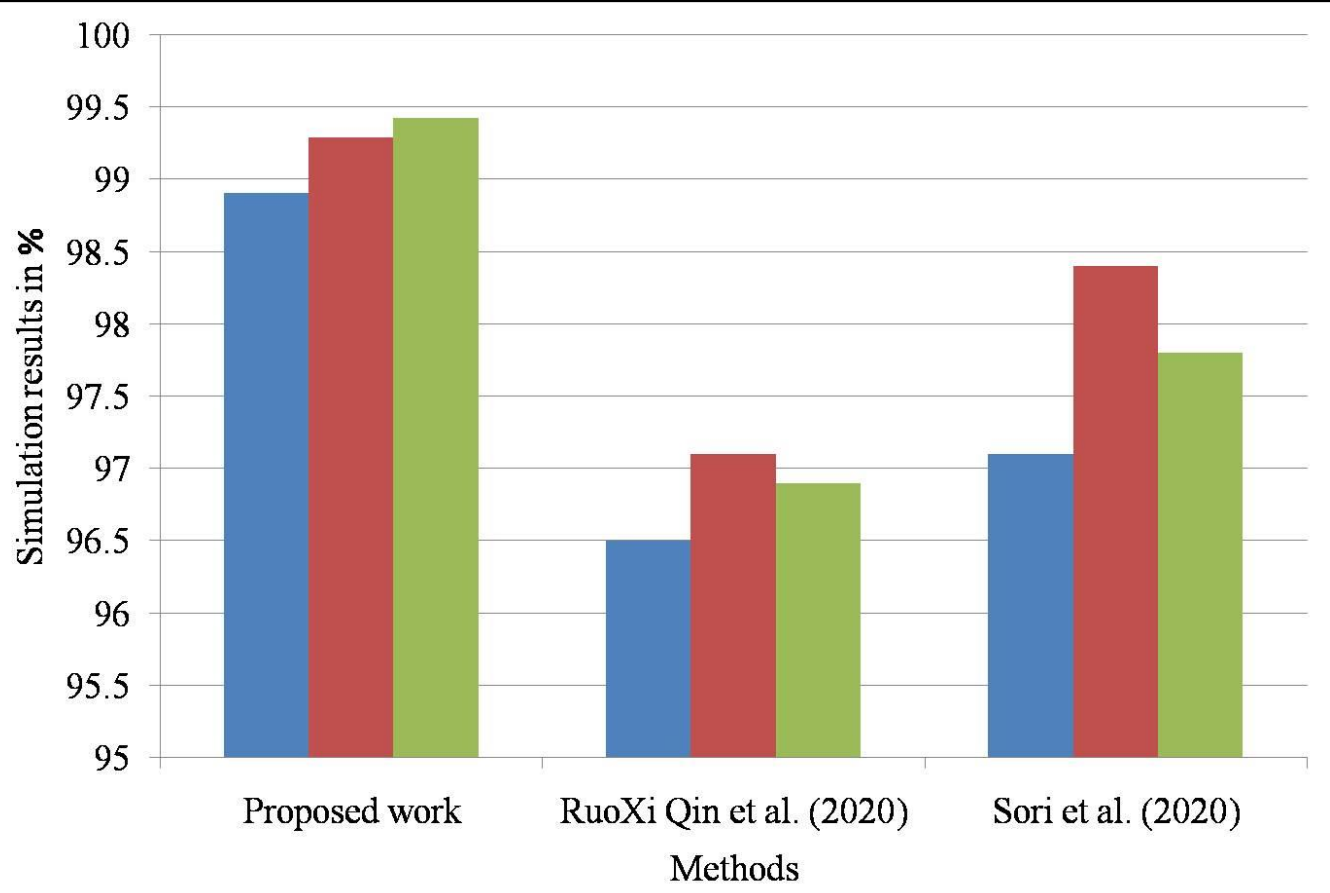

\section{$\square$ Average Diagnosis Index $\quad \square$ Segmentation accuracy $\quad$ Sensitivity}

\section{Conclusions}

Figure 7 Illustrations of the comparisons of simulation results

In this article, the tumors are detected in lung CT images using Convolutional Neural Networks (CNN) architecture with the help of data augmentation methods. TDIM for mild lung images is about $99 \%$ and the TDIS for severe lung images is about $98.8 \%$ in this article. The proposed lung CT tumor detection system achieves $98.9 \%$ of average diagnosis index, $99.29 \%$ of segmentation accuracy and $99.42 \%$ of sensitivity. In future, CNN architecture will be used to detect the abnormality in lung PET images.

\section{References}

1. Havaei M, Davy A, Warde-Farley D, Biard A, Courville A, Bengio Y, Pal C, Jodoin P M, Larochelle H. Brain tumor segmentation with deep neural networks. Medical Image Analysis, 2017, 35:18-31.

2. Jifara W, Jiang F, Rho S, Cheng M, Liu S. Medical image denoising using convolutional neural netwok: a residual learning approach. Journal of Super Computing, 2019, 75(2): 704-718.

3. Ren S, He K, Girshick R, Sun J. Faster R-CNN: towards real-time object detection with region proposal networks. In: Proceedings of Advances in Neural Information Processing Systems. 2015, 91-99.

4. Jiang X, Pang Y, Sun M, Li X. Cascaded sub patch networks for effective cnns. IEEE Transactions on Neural Networks and Learning Systems, 2017, 29(7): 2684-2694.

5. RuoXi Qin, Zhenzhen Wang, Ling Yun Jiang, Kai Qiao, Jinjin Hai, Jian Chen, Junling Xu, Dapeng Shi, Bin Yan, "Fine-Grained Lung Cancer Classification from PET and CT Images Based on Multidimensional Attention Mechanism", Complexity, vol. 2020, Article ID 6153657, 12 pages, 2020.

6. Sori, W.J., Feng, J., Godana, A.W. et al. DFD-Net: lung cancer detection from denoised CT scan image using deep learning. Front. Comput. Sci. 15, 152701 (2020).

7. W. Sun, B. Zheng, and Q. Wei, "Computer aided lung cancer diagnosis with deep learning algorithms," in Proceedings of the Medical Imaging: Computer-Aided Diagnosis, vol. 9785, p. 97850Z, San Diego, CA, USA, March 2016.

8. Teramoto, T. Tsukamoto, Y. Kiriyama, and H. Fujita, "Automated classification of lung cancer types from cytological images using deep convolutional neural networks," BioMed Research International, vol. 2017, Article ID 4956063, 9 pages, 2017.

9. S. Lakshmanaprabu, S. N. Mohanty, K. Shankar, N. Arunkumar, and G. Ramirez, "Optimal deep learning model for classification of lung cancer on CT images," Future Generation Computer Systems, vol. 92, pp. 374-382, 2019.

10. T. A. Jemimma and Y. J. Vetharaj, "Watershed Algorithm based DAPP features for Brain Tumor Segmentation and Classification," 2018 International Conference on Smart Systems and Inventive Technology (ICSSIT), Tirunelveli, India, 2018, pp. 155-158.

11.https://imaging.cancer.gov/informatics/lidc_idri.htm 